\title{
Photo-induced Functionalization on Biomaterials Surfaces
}

\author{
Kazuhiko Ishihara and Masayuki Kyomoto \\ Department of Materials Engineering, Department of Bioengineering, \\ 7-3-1, Hongo, Bunkyo-ku, Tokyo 113-8656, Japan \\ and \\ Core Research Evolutional Science and Technology (CREST) \\ Japan Science and Technology Agency (JST) \\ 5 Sanban-cho, Chiyoda-ku, Tokyo 102-0075, Japan \\ ishihara@mpc.t.u-tokyo.ac.jp
}

\begin{abstract}
We reported the fabrication of a highly hydrophilic nanometer-scale modified surface on the inert polymer substrate by photo-induced graft polymerization of 2-methacryloyloxyethyl phosphorylcholine (MPC) to obtain biocompatible surfaces. As substrates, poly(ether-ether-ketone) (PEEK) were used. Photoinduced radical formation on the substrate could be realized without any photosensitizer because of the benzophenone units in PEEK molecular structure, which acts as a photo-initiator during the graft polymerization. The poly(MPC)(PMPC)-grafted PEEK surface fabricated by a novel and simple polymerization system exhibited unique characteristics such as high wettability and high anti-protein adsorption, which makes it highly suitable for medical applications.

Keywords: poly(ether-ether-ketone), self-initiated photopolymerization, biomaterials
\end{abstract}

\section{Introduction}

Surface modification is one of the most important technologies for the preparation of new multifunctional biomaterials for satisfying several requirements; surface modifications used today include coating, blending, and grafting. In general, graft polymerization is performed most frequently using either of the following methods that utilize chemical and/or physical processes: (a) surface-initiated graft polymerization or "grafting from" methods in which the monomers are polymerized from initiators or comonomers, and (b) adsorption of the polymer to the substrate or "grafting to" methods, such as reaction of the end groups of the ready-made polymers with the functional groups of the substrate. The "grafting from" method has an advantage over the "grafting to" method wherein it forms a high-density polymer brush interface with a multifunctional polymer; this advantage proves to be functionally effective.

When the benzophenene (BP) is exposed to photo-irradiation such as ultraviolet-ray (UV)-irradiation, a pinacolization reaction is induced; this results in the formation of semi-benzopinacol radicals (i.e., ketyl radicals) that act as photo-initiators. Therefore, in this study, we have focused upon a BP unit in PEEK and formulated a self-initiated surface-graft polymerization method that utilizes the BP unit in graft-from polymerization (Fig. 1).

2-Methacryloyloxyethyl phosphorylcholine (MPC), a methacrylate monomer bearing a phosphorylcholine group, is used to synthesize polymer biomaterials having excellent biocompatibility [1-3]; the MPC polymers have potential applications in a variety of fields such as biomedical science, surface science, and

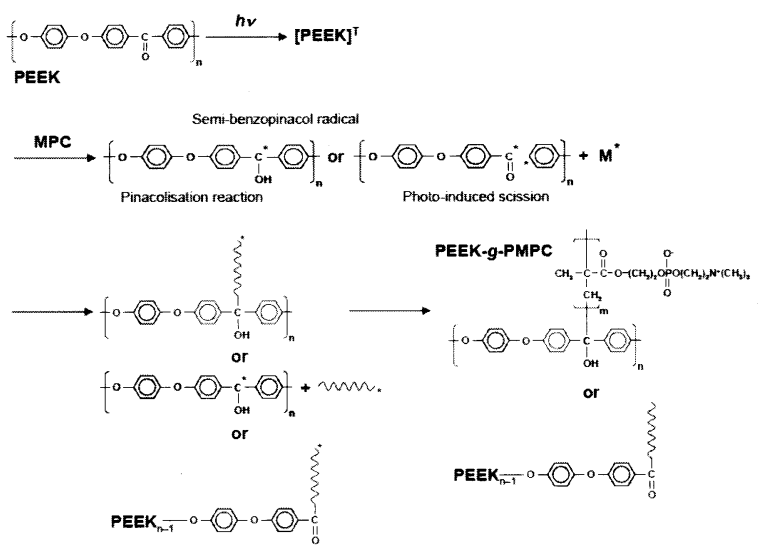

Fig. 1 Scheme for photo-induced polymerization of MPC on PEEK substrate. 
bioengineering because they possess unique properties such as excellent antibiofouling, and low friction abilities. Thus, surface modification with the MPC polymer on medical devices is effective for obtaining biocompatibility.

In this review, we have demonstrated the fabrication of a biocompatible and highly hydrophilic nanometer-scale-modified surface by poly(MPC) (PMPC)-grafting onto the self-initiated PEEK surface using a photo-induced reaction; further, we also investigated the effects of photo-irradiation time and MPC concentration variability on PMPC-graft polymerization $[4,5]$. The results revealed that it was possible to control the PMPC-graft layer in order to improve wettability, lubricity, and anti-protein adsorption for developing multifunctional PEEK biomaterials.

\section{Materials and methods}

\subsection{Self-initiated Graft Polymerization of MPC}

The preparation of PMPC-grafted PEEK is schematically illustrated in Fig. 1. PEEK specimens were machined from an extruded PEEK (450G; Victrex PLC, Thornton-Cleveleys, UK) bar stock, which was fabricated without stabilizers or additives. The surfaces of the PEEK specimens were ultrasonically cleaned in ethanol for $20 \mathrm{~min}$, and then dried in vacuum. MPC was synthesized using the method reported by Ishihara et al. [6]. It was dissolved in degassed water to obtain 0.25 - and $0.50-\mathrm{mol} / \mathrm{L}$ MPC aqueous solutions, and PEEK specimens were immersed in these solutions. Photo-induced graft polymerization was carried out at $60^{\circ} \mathrm{C}$ for $5-90$ min on the PEEK surface under UV-irradiation (UVL-400HA ultra-high pressure mercury lamp; Riko-Kagaku Sangyo Co. Ltd., Funabashi, Japan) with an intensity of $5 \mathrm{~mW} / \mathrm{cm}^{2}$; a filter (model D-35; Toshiba Corp., Tokyo, Japan) was used to restrict the passage of UV-light to wavelengths of $350 \pm 50 \mathrm{~nm}$. After polymerization, the PMPC-grafted PEEK specimens were removed from the reaction solution, washed with pure water and ethanol to remove non-reacted monomers and non-grafted polymers, and dried at room temperature.

\subsection{Surface Analysis of PMPC-grafted PEEK}

The functional group vibrations of the PMPC-grafted PEEK surfaces were examined by Fourier-transform infrared (FT-IR) spectroscopy with attenuated total reflection (ATR) equipment. The FT-IR/ATR spectra were obtained using an FT-IR analyzer (FT/IR615; JASCO Co. Ltd., Tokyo,
Japan) for 32 scans over the range of 1000-1800 $\mathrm{cm}^{-1}$ at a resolution of $4.0 \mathrm{~cm}^{-1}$.

The surface elemental conditions of the PMPC-grafted PEEK surfaces were analyzed by X-ray photoelectron spectroscopy (XPS). The XPS spectra were obtained using an XPS spectrophotometer (AXIS-HSi165; Kratos/ Shimadzu Co., Kyoto, Japan) equipped with a $15-\mathrm{kV} \mathrm{Mg-Ka}$ radiation source at the anode. The take-off angle of the photoelectrons was maintained at $90^{\circ}$. Five scans were taken for each sample.

The static-water contact angles on the PMPC-grafted PEEK surfaces were measured by the sessile drop method using an optical bench-type contact angle goniometer (Model DM300; Kyowa Interface Science Co. Ltd., Saitama, Japan). Measurements were repeated fifteen times for each sample, and the average values were regarded as the contact angles.

\subsection{Mechanical Test}

The mechanical properties of untreated PEEK and PMPC-grafted PEEK with a $0.50-\mathrm{mol} / \mathrm{L}$ MPC concentration and 90-min photo-irradiation time were evaluated with tensile and flexural tests. Tensile testing was performed according to ISO527 standard using a type 1B tensile bar specimen and a crosshead speed of $50 \mathrm{~mm} / \mathrm{min}$. Flexural testing was performed according to ISO178 standard with a crosshead speed of $2 \mathrm{~mm} / \mathrm{min}$. The results derived from each measurement in the mechanical test were expressed as the mean values \pm standard deviation. The statistical significance $(p<0.05)$ was estimated by Student's $t$-test.

\subsection{Characterization of Protein Adsorption by} Micro-bicinchoninic Acid Method

The amount of protein adsorbed on the PMPC-grafted PEEK surfaces was measured by the micro-bicinchoninic acid (BCA) method. Each specimen was immersed in Dulbecco's phosphate-buffered saline (PBS, $\mathrm{pH}$ 7.4, ion strength $=0.15 \mathrm{M}$; Immuno-Biological Laboratories Co. Ltd., Takasaki, Japan) for $1 \mathrm{~h}$ to equilibrate the PMPC-grafted surface. The specimens were immersed in bovine serum albumin (BSA, $\mathrm{Mw}=6.7$ $\times 10^{4}$; Sigma-Aldrich Corp., MO, USA) solution at $37^{\circ} \mathrm{C}$ for $1 \mathrm{~h}$. The protein solution was prepared in a BSA concentration of $4.5 \mathrm{~g} / \mathrm{L}$, i.e., $10 \%$ of the concentration of human plasma levels. Then, the specimens were rinsed five times with fresh PBS and immersed in $10.0-\mathrm{g} / \mathrm{L}$ sodium dodecyl sulfate (SDS) aqueous solution and shaken at room 
temperature for $1 \mathrm{~h}$ to completely detach the adsorbed BSA on the PMPC-grafted surface. A protein analysis kit (micro BCA protein assay kit, \#23235; Thermo Fisher Scientific Inc., IL, USA) based on the BCA method was used to determine the BSA concentration in the SDS solution, and the amount of BSA adsorbed on the PMPC-grafted PEEK surface was calculated.

\section{Results}

FT-IR/ATR spectra of untreated PEEK and PMPC-grafted PEEK with a $0.50-\mathrm{mol} / \mathrm{L}$ MPC concentration and 90-min photo-irradiation time shows transmission absorption peaks were observed at $1600,1490,1280,1190$, and $1160 \mathrm{~cm}^{-1}$ for both untreated PEEK and PMPC-grafted PEEK. These peaks are chiefly attributed to the diphenyl ether group, phenyl rings, or aromatic hydrogens in the PEEK substrate [7]. However, absorption peaks at 1720 and $1080 \mathrm{~cm}^{-1}$ (shoulder peak) were observed only for PMPC-grafted PEEK. These peaks corresponded to the carbonyl group $(\mathrm{C}=\mathrm{O})$ and phosphate group $(\mathrm{P}-\mathrm{O})$ in the MPC unit, respectively [8]. The XPS spectra of the binding energy region of the nitrogen $(\mathrm{N})$ and phosphorous $(\mathrm{P})$ electrons showed peaks for untreated PEEK and PMPC-grafted PEEK, whereas, peaks were not

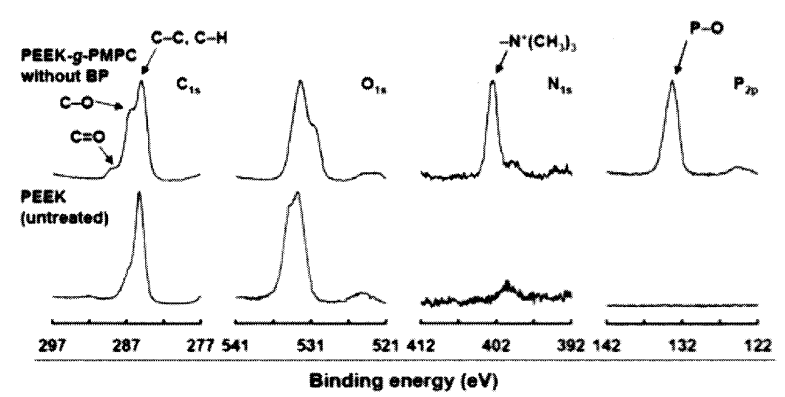

Fig. 2. XPS spectra of PEEK and PMPC-grafted PEEK.

observed in the case of untreated PEEK. The peaks at 403 and $134 \mathrm{eV}$ were attributed to the $-\mathrm{N}^{+}\left(\mathrm{CH}_{3}\right)_{3}$ and phosphate groups, respectively. These peaks indicate the presence of phosphorylcholine in the MPC units. After PMPC grafting, the peaks attributed to the MPC unit were clearly observed in both the FT-IR/ATR and XPS spectra of the PMPC-grafted PEEK. These results indicate that PMPC was successfully grafted on the PEEK surface.

Fig. 3 shows the $\mathrm{N}$ and $\mathrm{P}$ concentrations of the PMPC-grafted PEEK as a function of the photo-irradiation time during polymerization at
0.25- and 0.50-mol/L MPC concentrations. The N and $\mathrm{P}$ concentrations increased with the photo-irradiation time. When the photo-irradiation time was shorter than $45 \mathrm{~min}$, the $\mathrm{N}$ and $\mathrm{P}$ concentrations of the PMPC-grafted PEEK surface with a $0.50-\mathrm{mol} / \mathrm{L}$ MPC concentration were higher as compared with those with a $0.25-\mathrm{mol} / \mathrm{L} \mathrm{MPC}$ concentration. The $\mathrm{N}$ and $\mathrm{P}$ concentration in the PMPC-grafted PEEK with both $0.25-$ and 0.50-mol/L MPC concentrations increased to 5.2 up to a photo-irradiation time of $90 \mathrm{~min}$; those values were almost equivalent to the theoretical elemental composition $(\mathrm{N}=5.3, \mathrm{P}=5.3)$ of PMPC. These results indicate that the PMPC layer fully covered the surface of the PEEK substrate.

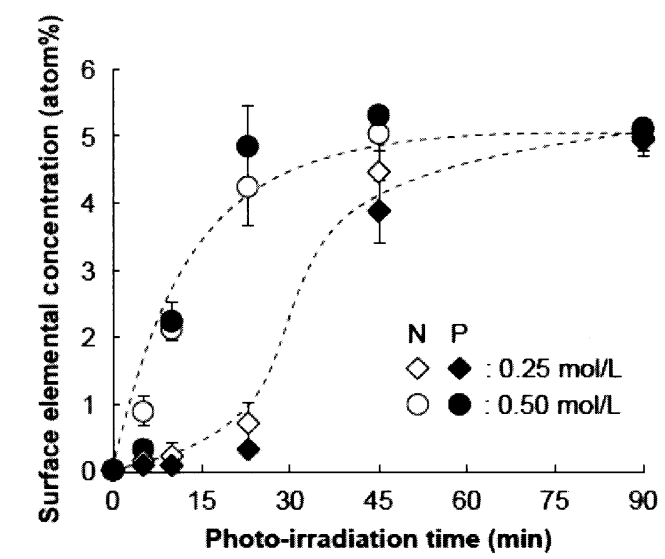

Fig. 3. Surface elemental concentration on PMPC-graft PEEK as a function of photo-irradiation time.

The physical properties of PMPC-graft layer on the PEEK surface as a function of the photo-irradiation time at MPC concentrations of 0.25 and $0.50 \mathrm{~mol} / \mathrm{L}$ was evaluated. The PMPC-graft layer density of the PMPC-grafted PEEK with $0.25-\mathrm{mol} / \mathrm{L}$ MPC concentrations increased proportionally to $2.3 \mathrm{~g} / \mathrm{cm}^{3}$ with the photo-irradiation time. In the case of PMPC-grafted PEEK obtained with $0.50-\mathrm{mol} / \mathrm{L}$ MPC concentrations, the PMPC-graft layer density rapidly increased to $1.3 \mathrm{~g} / \mathrm{cm}^{3}$ up to a photo-irradiation time of $10 \mathrm{~min}$; it then increased slowly to $2.2 \mathrm{~g} / \mathrm{cm}^{3}$ with an increase in the photo-irradiation time.

The mechanical properties of untreated PEEK and PMPC-grafted PEEK are summarized in Table 1. Tensile properties and flexural modulus did not differ significantly $(p>0.05)$ between untreated PEEK and PMPC-grafted PEEK. In contrast, there was a small but significant difference $(p<0.05)$ in the flexural strength and strain of untreated PEEK 
Table 1. Mechanical property of PMPC-grafted PEEK.

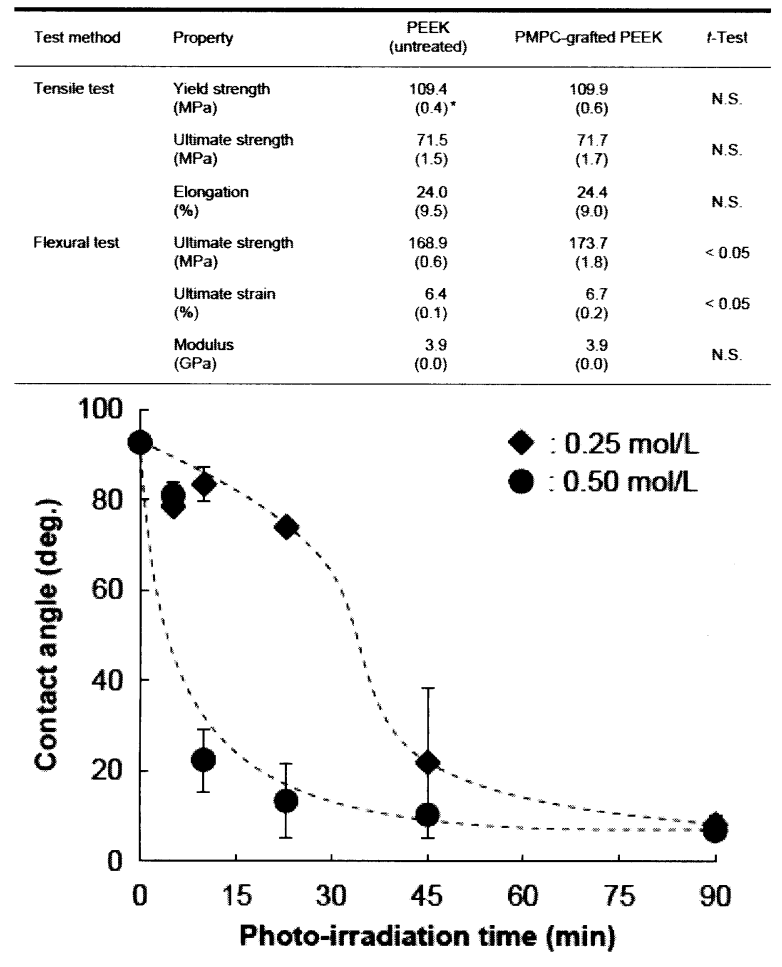

Fig. 4. Static contact angle by water on PMPC-grafted PEEK as a function of photo-irradiation time.

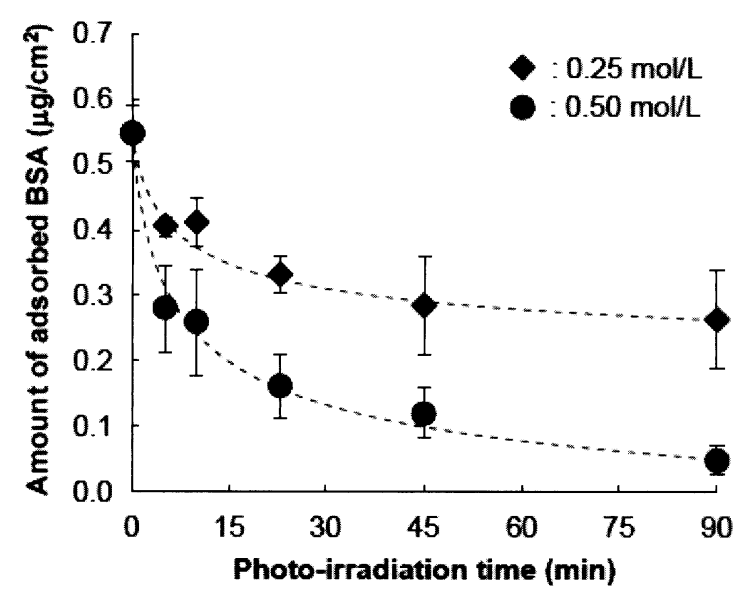

Fig. 5. Protein adsorption on PMPC-grafted PEEK as a function of photo-irradiation time.

and PMPC-grafted PEEK examined in this study. However, both untreated PEEK and PMPC-grafted PEEK met the ASTM F2026 requirements.

Fig. 4 shows the static-water contact angle of PMPC-grafted PEEK obtained with MPC concentrations of 0.25 and $0.50 \mathrm{~mol} / \mathrm{L}$ as a function of the photo-irradiation time. The static-water contact angle of untreated PEEK was $90^{\circ}$ and decreased markedly with an increase in the photo-irradiation time. When the photo-irradiation time was $90 \mathrm{~min}$, the static-water contact angle of
PMPC-grafted PEEK was the lowest value at $<10^{\circ}$.

Fig. 5 shows the amount of protein (BSA) adsorbed on PMPC-grafted PEEK as a function of the photo-irradiation time with MPC concentrations of $0.25 \mathrm{~mol} / \mathrm{L}$ and $0.50 \mathrm{~mol} / \mathrm{L}$. The amount of adsorbed BSA of PMPC-grafted PEEK decreased remarkably with an increase in photo-irradiation time.

\section{Discussion}

We demonstrated the fabrication of a biocompatible and highly hydrophilic nanometer-scale-modified surface by PMPC-grafting onto the self-initiated PEEK surface using a photo-induced grafting-from polymerization reaction, i.e., "self-initiated surface graft polymerization." The following methods were employed in our study: (a) grafting from polymerization for the formation of a high-density graft polymer layer, (b) photo-induced polymerization in the absence of photo-initiators, and (c) using biocompatible hydrophilic macromolecules, which exhibited photo-reduction by $\mathrm{H}$-abstraction of a BP unit in PEEK from an H-donor; this induced surface-initiated graft polymerization of the feed methacrylate-type monomer (i.e., MPC) on the PEEK surface, even in the absence of a photo-initiator such as BP.

It is important to control the thickness and density of graft polymer layer for optimizing several areas of applications, e.g., in adhesion, colloid stabilization, and lubrication. When the photo-irradiation time was greater than $45 \mathrm{~min}$, the PMPC-graft layer thicknesses became almost constant. Moreover, at the same photo-irradiation time of $90 \mathrm{~min}$, it was shown that the higher the monomer concentration, the thicker the graft layer obtained: the PMPC-graft layer thickness (approximately $100 \mathrm{~nm}$ ) of the PMPC-grafted PEEK obtained with $0.50-\mathrm{mol} / \mathrm{L}$ MPC concentration was thicker than that (approximately $40 \mathrm{~nm}$ ) with $0.25-\mathrm{mol} / \mathrm{L}$ MPC concentration. The phenomenon can be attributed to the fact that the graft layer thickness increases with monomer concentration. When the PMPC layer has a brush-like structure, the graft layer thickness may correlate with the molecular weight of the grafted PMPC. The high-density PMPC-graft layer on the PEEK surface is assumed to exhibit a brush-like structure $[9,10]$. It is generally well known that the reaction rate of radical polymerization is extremely high [11]. It was observed that the graft layer thickness (i.e., molecular weight of the graft polymer) was greatly 
dependent on the monomer concentration, but virtually independent of the photo-irradiation time. Thus, in this study, the length (molecular weight) of the PMPC-graft chains was assumed to be successfully controlled by the MPC concentration used for polymerization. This indicates that the length of the PMPC chain grafted on the PEEK surface increased with the MPC concentration during polymerization [12]. Additionally, a uniform PMPC layer was clearly observed on the surface of the PEEK substrate, and no cracks or delaminating were observed at the PEEK substrate or the interface between the PMPC layer and PEEK substrate. These results indicate that the PMPC layer formed on the PEEK substrate is uniformly distributed over the substrate and is bound to the substrate by covalent $\mathrm{C}-\mathrm{C}$ bonds.

On the other hand, the PMPC-graft layer density on the PEEK surface almost linearly increased to $>2.2 \mathrm{~g} / \mathrm{cm}^{3}$ with the photo-irradiation time, suggesting that the graft chain propagates steadily with increasing photo-irradiation time. In order to obtain the high-density PMPC-graft layer, the photo-irradiation time must be controlled. Further, interestingly, while using an MPC concentration of $0.50 \mathrm{~mol} / \mathrm{L}$, the rate of the increase in the PMPC-graft layer density was low with a photo-irradiation time above $10 \mathrm{~min}$. The present self-initiated surface graft polymerization method is photo-induced by UV-irradiation onto the BP unit of PEEK surface. In this study, it is assumed that the UV-irradiation directly produces a high-concentration free radical, because this PEEK is a semi-crystalline structure (crystalline, 30-40\%) with a high-density BP unit in the surface. When the MPC concentration in a feed is also high, the self-initiated surface graft polymerization between the radicals on the PEEK surface and the MPC occurs extremely rapidly in the reaction system, forming the high-density graft chain on the surface. Hence, diffusion of the MPC monomer onto the PEEK surface might be interfered by the high-density graft chain because of its high viscosity. When the monomers attached to the PEEK surface were subjected to UV-irradiation, radicals were freely formed on the PEEK surface in the early stage but not in the late stage of polymerization, probably because the high-density grafted polymer chains formed by then blocked the diffusion of the monomer to the PEEK surface. Therefore, it is supposed that the rate of increase in the PMPC-graft layer density changed due to the high concentration of free radicals and monomers.
The wettability by water of the PMPC-grafted PEEK surface is considerably greater than that of the untreated PEEK surface (Fig. 4), because of the presence of a nanometer-scaled PMPC layer: MPC is a highly hydrophilic compound, while PMPC is water-soluble. We also observe that the dynamic coefficient of friction was greatly dependent on the water-wettability (static-water contact angle), but virtually independent of the structure (thickness and density) of the graft layer. A significant reduction in the static-water contact angle of the PMPC-grafted surface resulted in a substantial improvement in friction property. Fluid-film lubrication (or hydration lubrication) with the PMPC-grafted surface was achieved by the intermediate hydrated layer. It can be affirmed that this highly lubricated surface utilizing PMPC mimics the natural cartilage structure [13]. When the PEEK surface is modified by PMPC-grafting, the grafted PMPC causes a significant reduction in sliding friction between the graft surfaces because the thin water films that are formed act as extremely efficient lubricants. The water-lubrication systems utilizing PMPC suppress direct contact of the counter-bearing face with the PEEK substrate in order to reduce the frictional force. Thus, the PMPC-graft layer is expected to significantly increase the durability of the bearing biomaterials.

The fabrication of surfaces that exhibit anti-protein adsorption and/or cell adhesion has been one of goals of surface engineering for medical devices. The adsorption of the BSA on the PMPC-grafted PEEK surface decreased to $10 \%(p<$ 0.001 ) with an increase in photo-irradiation time, as shown in Fig. 5, as compared to that in untreated PEEK. It is shown that the extent of protein rejection is related to the PMPC-graft extent. Extensive grafting gives rise not only to an increase in the thickness of graft layer but also to an increase in the volume fraction (i.e., density) of graft segments in the layer. Therefore, it was thought that these characteristics of thickness and density of the PMPC-graft layer had a significant influence on protein adsorption. The mechanism of protein adsorption resistance on the PMPC-grafted surface is hypothesized as follows: the protein adsorption resistance attributed to the water-fluid film and hydration layer was due to water and hydrophilic macromolecules with volume exclusion effects. The presence of the water-fluid film and hydration layer is responsible for the easy detachment of proteins and the prevention of conformational changes in the adsorbed proteins. These results 
imply that the PMPC-grafted PEEK surface is biocompatible in terms of tissue and blood compatibility, because MPC polymer-modified surfaces are known to exhibit in vivo antibiofouling.

As shown in Table 1, the mechanical properties of the PEEK are unchanged even after PMPC-grafting. This indicates that the photo-induced radical graft polymerization proceeds only on the surface of the PEEK substrate, while the properties of the substrate remain unchanged. Retention of the properties of the PEEK substrate is very important in clinical use, because the biomaterials used in implants act not only as surface-functional materials but also as structural materials in vivo.

The design of a well-characterized surface is a considerably important and difficult task. The photo-induced graft polymerization in the absence of photo-initiators of this study, i.e., "self-initiated surface graft polymerization" successfully prepared the surface with controlled graft layer thickness and density. This simple self-initiated surface graft polymerization would be highly suitable for industrial applications as well as the development of medical devices. The synthesis of a self-initiated biocompatible polymer having unique properties such as anti-protein adsorption and wettability by the photoinduced grafting-from polymerization reaction is a novel phenomenon in the field of biomaterials and bioengineering sciences, and the fabrication of the PMPC-grafted PEEK surface can result in the development of next-generation multifunctional biomaterials.

\section{Conclusions}

A biocompatible and highly hydrophilic nanometer-scale modified surface was successfully fabricated on the PEEK substrate by the photo-induced graft polymerization of PMPC in the absence of photo-initiators, i.e., "self-initiated surface graft polymerization." Since MPC is a highly hydrophilic compound, the water wettability and lubricity of the PMPC-grafted PEEK surface were greater than that of the untreated PEEK surface due to the formation of a PMPC nanometer-scale layer. In addition, the amount of BSA adsorbed on the PMPC-grafted PEEK surface considerably decreased compared to that in the case of untreated PEEK. The density and thickness of the grafting layer could be controlled by the photo-irradiation time and monomer concentration.

\section{References}

1. Ishihara $\mathrm{K}$, Nomura $\mathrm{H}$, Mihara $\mathrm{T}$, Kurita $\mathrm{K}$, Iwasaki Y, Nakabayashi N, J Biomed Mater Res 1998, 39, 323-330.

2. Ishihara K, Ziats NP, Tierney BP, Nakabayashi N, Anderson JM, J Biomed Mater Res 1991, 25, 1397-1407.

3. Ishihara $\mathrm{K}$, Oshida $\mathrm{H}$, Ueda T, Endo Y, Watanabe A, Nakabayashi N, J Biomed Mater Res 1992, 26, 1543-1552.

4. Kyomoto M, Ishihara K, ACS Appl Mater Interfaces 2009, 1, 537-542.

5. Kyomoto M, Moro T, Takatoti Y, Kawaguchi H, Nakamura K, Ishihara K, Biomaterials 2010, 31, 1017-1024.

6. Ishihara K, Ueda T, Nakabayashi N, Polym J 1990, 22, 355-360.

7. He D, Susanto H, Ulbricht M, Prog Polym Sci 2009, 34, 62-98.

8. Kyomoto M, Moro T, Konno T, Takamada $\mathrm{H}$, Yamawaki N, Kawaguchi H, Nakamura K, Ishihara $\mathrm{K}, J$ Biomed Mater Res A 2007, 82, 10-17.

9. Kyomoto M, Moro T, Iwasaki Y, Miyaji F, Kawaguchi H, Takatori Y, Nakamura K, Ishihara K, J Biomed Mater Res A 2009, 91, 730-741.

10. Matsuda T, Kaneko M, Ge S, Biomaterials 2003, 24, 4507-4515.

11. Braunecker WA, Matyjaszewsky K, Prog Polym Sci 2007, 32, 93-146.

12. Kyomoto M, Moro T, Miyaji F, Hashimoto M, Kawaguchi H, Takatori Y, Nakamura K, Ishihara K, J Biomed Mater Res A 2008, 86, 439-447.

13. Ishikawa Y, Hiratsuka K, Sasada T, Wear 2006, 261, 500-505. 